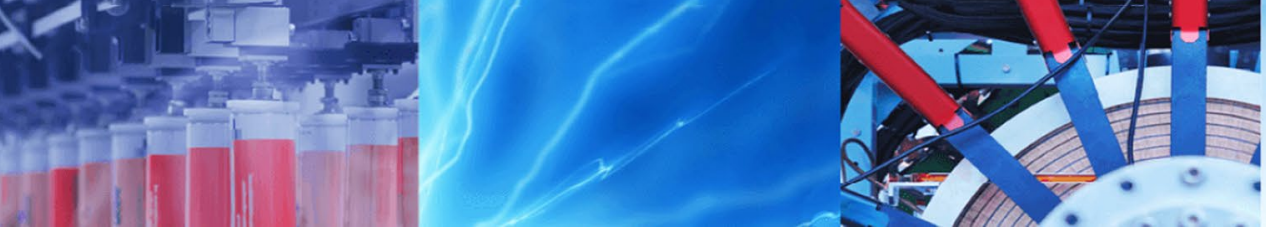

Research Article

\title{
A general theory for coupled chemo-electro-thermo-mechanical heterogeneous system
}

\author{
Zhen-Bang Kuang ${ }^{1}$ (I)
}

Received: 7 April 2020 / Accepted: 4 November 2020 / Published online: 12 December 2020

(c) Springer Nature Switzerland AG 2020

\begin{abstract}
Many transport and rate processes in chemical, physical, mineral, material and biological fields are controlled by the coupled chemo-electro-thermo-mechanical (CETM) process. Though many literatures discussed these coupled problems, but a unified rigorous theory and a unified method based on the chemical thermodynamics are lacked. In this paper on the basis of electrochemistry, the non-equilibrium thermodynamics and modern continuum mechanics we modify some previous theories and give a general theory including mass conservation equation, the electric charge conservation equation, complete energy conservation equation, entropy equation, evolution equations and the complete governing equations of these couple CETM systems. An extension of Nernst-Planck equation is derived for the CETM system. This theory gives a theoretical foundation and a universal method to improve and develop engineering theories, especially for the gradual failure components and cells. In appendix we also discuss the interdiffusion problems in solids with vacancies shortly as a complement of the continuum diffusion.
\end{abstract}

Keywords Energy equation · Chemo-electro-thermo-mechanical systems · Entropy equation and entropy production rate - Gibbs equation - Evolution equation - Governing equation

\section{Introduction}

In chemical, physical, material and biological systems, many transport and rate processes are controlled by the coupled chemo-electro-thermo-mechanical interaction. We shall abbreviate a system with coupled Chemo-Electro-Thermo-Mechanical interaction as CETM system. Similarly, a system with coupled Chemo-Thermo-Mechanical interaction is a CTM system and a system with coupled Chemo-Electro-Mechanical interaction is a CEM system. All these systems are complex thermodynamic systems. A general theory for CTM system has been discussed in papers [1, 2], where a complete energy conservation equation, an appropriate entropy equation and the governing equation system have been given and modified the previous theories [3-8]. Most of these previous theories are for electrically neutral system. However, in engineering a system may be worked under electromagnetic field, which may be externally imposed or internally created, or both. In a thermoelectric material there exist Seebeck, Peltier and Thomson phenomena [3, 9]. Ionic and mixed ionic-electronic devices, such as solid oxide fuel cells, oxygen pumps and hydrogen production, have gained many applications [10-13]. For expansive media including clays, shales, polymers gels, corneal endothelium, immature articular cartilage and connective biological tissues, electrochemical interaction are also typical [14-16]. In theories of these chemo-electro-mechanical (CEM) system the temperature effects are neglected [9-16]. Especially when one discusses the gradual failure of components and cells the temperature effects may be important. So integrating the effects of the electromagnetic fields into the CTM system

Zhen-Bang Kuang, zbkuang@sjtu.edu.cn | 'School of Naval Architecture, Ocean and Civil Engineering, Shanghai Jiaotong University, Shanghai 200240, China. 
and integrating the effects of the temperature fields into the CEM system are necessary. Though in [3] the CETM system without chemical reaction had been discussed, but its results are still left improvement. Therefore the CETM systems are worth to study. For a CETM system the mass equation, energy equation, entropy equation and momentum equation can be studied unitedly, but the electric fields are produced due to various reasons and every case should be researched independently. This situation is analogous to the forces in the mechanical action. The complete coupling effects of heat, diffusion, chemical effect and electromagnetic field are fully considered on a unified thermodynamic foundation and a theoretical frame of governing equations are given. An appropriate complete governing equation system is the foundation for solving engineering problems. The electrochemistry, the non-equilibrium thermodynamics and modern continuum mechanics allow us to construct an efficient theory for this coupling problems.

We discuss an open system consisted of $N$ (charged or uncharged) species with total mass $M$, total volume $V$ and total density $\rho$. According to the engineering custom we use the partial density or the mass concentration $\rho^{(k)}=M^{(k)} / V$ of a species $k$, rather than the molar concentration $C^{(k)}=\rho^{(k)} / M_{M}^{(k)}$, where $M^{(k)}$ and $M_{M}^{(k)}$, are the total mass in $V$ and the molecular weight of the species $k$ respectively.

\section{The mass and electric charge conservation equations}

\subsection{The mass conservation equation}

The mass conservation equation of the species $k$ is the same for CETM and CTM systems because the macroscopic mass is independent to the electromagnetic field. For easy to read the mass conservation equation given in literatures [1-4] is repeated here.

Let a CETM system be consisted of $N=N_{1}+N_{2}$ species with $N_{1}$ reactants and $N_{2}$ products, the total mass $M$, total volume $V$, total density $\rho$ and chemical reaction number $L$. The $j^{\text {th }}$ chemical reaction equation can be written as $[1$, 2, 17-20]

$$
\sum_{k=1}^{N} \nu^{(j k)} B^{(k)}=0, \quad j=1,2 \ldots, L
$$

where $B^{(k)}$ is the chemical formula of species $k, v^{(j k)}$ is the stoichiometric constant of a species $k$ in the chemical reaction $j$, which is positive if species $k$ is a product and negative if $k$ is a reactant. If $B^{(m)}$ is not appear in the $j^{\text {th }}$ chemical reaction, then $v^{(\mathrm{jm})}=0$. In this paper, we use superscript to denote the serial number of the species and the serial number of the chemical reaction and the summation notation is written in evidence, as shown in Eq. (1); but the subscripts are used to components of a vector or tensor and the summation rule for the repeated indices is used.

Let $\rho^{(k)}, c^{(k)}, \boldsymbol{v}^{(k)}, v^{(j k)}, v^{(j k)} \dot{\xi}^{(j)}$ and $\dot{\Theta}^{(k)}$ be the partial density or the apparent density, the mass fraction, the velocity, the reaction rate, the mass production rate per volume in $j^{\text {th }}$ chemical reaction and in all chemical reactions of the species $k$ respectively; $\boldsymbol{v}=\dot{\boldsymbol{u}}$ and $\boldsymbol{u}$ are the barycentric velocity and the mechanical displacement vector of a representative element respectively, $\boldsymbol{J}^{(k)}$ is the mass diffusion flow of the species $k$. The mass conservation equation of the species $k$ is [1-4]:

$$
\begin{aligned}
& \rho \dot{c}^{(k)}=-\nabla \cdot \boldsymbol{J}^{(k)}+\dot{\Theta}^{(k)}, \quad()^{\cdot}=\frac{d()}{d t}=\frac{\partial()}{\partial t}+\boldsymbol{v} \cdot \nabla() \\
& \frac{\partial \rho^{(k)}}{\partial t}=-\nabla \cdot\left(\rho^{(k)} \boldsymbol{v}^{(k)}\right)+\dot{\Theta}^{(k)} ; \quad \dot{\rho}+\rho \nabla \cdot \boldsymbol{v}=0 \\
& c^{(k)}=\frac{\rho^{(k)}}{\rho}, \quad \rho=\sum_{k=1}^{N} \rho^{(k)}, \quad \sum_{k=1}^{N} c^{(k)}=1 \\
& \boldsymbol{J}^{(k)}=\rho^{(k)}\left(\boldsymbol{v}^{(k)}-\boldsymbol{v}\right), \quad \rho \boldsymbol{v}=\sum_{k=1}^{N} \rho^{(k)} \boldsymbol{v}^{(k)}, \quad \sum_{k=1}^{N} \boldsymbol{J}^{(k)}=\mathbf{0} \\
& \dot{\Theta}^{(k)}=\sum_{j=1}^{L} v^{(j k)} \dot{\xi}^{(j)}, \quad \sum_{k=1}^{N} \dot{\Theta}^{(k)}=0 ; \quad k=1,2, \cdots, N
\end{aligned}
$$

where $\nabla$ is the Eulerian gradient operator. Equation (2) shows that the mass fraction rate $\dot{c}$, the mass flow $\boldsymbol{J}^{(k)}$ and the total mass production rate $\dot{\Theta}^{(k)}$ of species $k$ subject to the equation

$$
\sum_{k=1}^{N} \dot{c}^{(k)}=0, \quad \sum_{k=1}^{N} \boldsymbol{J}^{(k)}=\mathbf{0}, \quad \sum_{k=1}^{N} \dot{\Theta}^{(k)}=0
$$

In the appendix, one will see that the interdiffusion in noncontinuum solids with vacancies [21,22] has different mechanism with the above theory and discussed shortly as the complement of the diffusion phenomena in continuum media.

\subsection{The electric charge conservation equation}

According to literature [3] one define

$$
\begin{aligned}
& \boldsymbol{I}=\sum_{k=1}^{N} \rho^{(k)} \boldsymbol{z}^{(k)} \mathbf{v}^{(k)}=\boldsymbol{I}_{0}+\boldsymbol{i} ; \quad \boldsymbol{I}_{0}=\rho z \boldsymbol{v}, \quad \boldsymbol{i}=\sum_{k=1}^{N} z^{(k)} \boldsymbol{J}^{(k)} \\
& \boldsymbol{z}=\rho^{-1} \sum_{k=1}^{N} \rho^{(k)} z^{(k)}=\sum_{k=1}^{N} c^{(k)} z^{(k)}
\end{aligned}
$$

where $z^{(k)}$ is the charge per mass of component $k, z$ is the total charge per mass of the system, $\boldsymbol{I}$ is the total electric 
current density, $\boldsymbol{I}_{0}$ is the convective current density and $\boldsymbol{i}$ is the conductive current density. Using Eq. (2) the electric charge conservation equation is

$\rho \dot{z}=\rho \sum_{k=1}^{N} \dot{c}^{(k)} z^{(k)}=\sum_{k=1}^{N}\left(-\nabla \cdot \boldsymbol{J}^{(k)}+\dot{\Theta}^{(k)}\right) z^{(k)}=-\nabla \cdot \boldsymbol{i}+\sum_{k=1}^{N} \dot{\Theta}^{(k)} z^{(k)}$

\section{The momentum and energy conservation equations in a CETM open system}

\subsection{The momentum conservation equation}

The electromagnetic force $\boldsymbol{f}^{(e m)}$ applied on a moving charge is given by Lorentz force law, so the momentum equation in CETM system is [23-26]

$\nabla \cdot \sigma+\rho\left(\boldsymbol{f}^{(m)}+\boldsymbol{f}^{(e m)}\right)=\rho \dot{\boldsymbol{v}}=\rho \ddot{\mathbf{u}}$,

$\rho \boldsymbol{f}^{(e m)}=\sum_{k=1}^{N} \rho^{(k)} z^{(k)}\left(\boldsymbol{E}+\boldsymbol{v}^{(k)} \times \boldsymbol{B}\right)=\rho z \boldsymbol{E}+\boldsymbol{I} \times \boldsymbol{B}$

where $\rho^{(k)} z^{(k)}\left(\boldsymbol{E}+\boldsymbol{V}^{(k)} \times \boldsymbol{B}\right)$ is the Lorentz force per volume acting on species $k, \rho \boldsymbol{f}^{(m)}$ is the mechanical body force per volume, $\sigma$ is the stress tensor of a representative element, $\boldsymbol{E}$ is electric field intensity, $\boldsymbol{B}$ is the magnetic induction.

From the Maxwell equation the more general momentum equation in CETM system for $|\boldsymbol{v}| \ll c$ is [23-26]

$\nabla \cdot\left(\sigma+\sigma^{M}\right)+\rho \boldsymbol{f}^{(m)}=\rho \dot{\boldsymbol{v}}$

$\boldsymbol{\sigma}^{M}=(\boldsymbol{D} \otimes \boldsymbol{E}+\boldsymbol{B} \otimes \boldsymbol{H})-(1 / 2)(\boldsymbol{D} \cdot \boldsymbol{E}+\boldsymbol{B} \cdot \boldsymbol{H}) \boldsymbol{\delta}$

where $\sigma^{M}$ is the Maxwell stress, $c$ is the light velocity. $\boldsymbol{D}$ is the electric displacement and $\boldsymbol{H}$ is the magnetic strength, $\delta$ is the unit tensor Kronecker delta, $\otimes$ is the tensor product and the component of $\boldsymbol{D} \otimes \boldsymbol{E}$ is $D_{i} E_{j}$. For linear uniform isotropic medium one can prove $\nabla \cdot \boldsymbol{\sigma}^{M}=\rho z \boldsymbol{E}+\boldsymbol{I} \times \boldsymbol{B}$.

\subsection{The energy conservation equation}

Multiplying Eq. (6) by $\boldsymbol{v}$ and using relations

$\boldsymbol{v} \cdot(\nabla \cdot \boldsymbol{\sigma})=\nabla \cdot(\boldsymbol{\sigma} \cdot \boldsymbol{v})-\boldsymbol{\sigma}:(\nabla \otimes \boldsymbol{v}) ; \quad \boldsymbol{\sigma}:(\nabla \otimes \boldsymbol{v})=\sigma_{i j} v_{i, j}$

$\boldsymbol{v} \cdot(\boldsymbol{I} \times \boldsymbol{B})=\boldsymbol{v} \cdot[(\rho z \boldsymbol{v}+\boldsymbol{i}) \times \boldsymbol{B}]=\boldsymbol{v} \cdot(\boldsymbol{i} \times \boldsymbol{B})=-\boldsymbol{i} \cdot(\boldsymbol{v} \times \boldsymbol{B})$

$\boldsymbol{v} \cdot(\rho z \boldsymbol{V} \times \boldsymbol{B})=0$

one get the kinetic energy rate $\dot{K}[3]$ :
From Eq. (9) it is known that a part of electromagnetic work, $\rho z \boldsymbol{E} \cdot \boldsymbol{v}-\boldsymbol{i} \cdot(\boldsymbol{V} \times \boldsymbol{B})$, is changed to the kinetic energy.

According to the Maxwell equation the balance law of electromagnetic energy [3, 23-26] is

$\frac{\partial \Phi}{\partial t}=\boldsymbol{E} \cdot \frac{\partial \boldsymbol{D}}{\partial t}+\boldsymbol{H} \cdot \frac{\partial \boldsymbol{B}}{\partial t}=-\nabla \cdot(\boldsymbol{E} \times \boldsymbol{H})-\boldsymbol{I} \cdot \boldsymbol{E}$

where $\Phi$ is the energy density stored in the electro-magnetic field, $\boldsymbol{E} \times \boldsymbol{H}$ is the Poynting vector of energy flow, $\boldsymbol{I} \cdot \boldsymbol{E}$ is the work on matter supplied by the electro-magnetic field. $\ln \boldsymbol{I} \cdot \boldsymbol{E}$ the part $\boldsymbol{i} \cdot \boldsymbol{E}$ produced by conductive current $\boldsymbol{i}$ is changed to Joule heat [26] and constitutes a part of the internal energy, but the part $\boldsymbol{I}_{0} \cdot \boldsymbol{E}$, produced by convective current $I_{0}$ which moves with the Centroid of medium, is not related to internal energy or Joule heat, but is changed to the kinetic energy of the system (see Eq. 9).

The energy equation in a CETM open system for a viscous fluid is

$\dot{U}+\dot{K}=\dot{W}+\dot{Q}+\dot{\Lambda}+\dot{I}$

$\dot{U}=\int_{V} \rho \dot{u} d V, \dot{K}=\int_{V} \rho \frac{1}{2}(\boldsymbol{v} \cdot \boldsymbol{v}) \cdot d V$

$\dot{W}=\int_{V} \rho\left(\boldsymbol{f}^{(m)}+\boldsymbol{f}^{(e m)}\right) \cdot \boldsymbol{v} d V+\int_{a} \boldsymbol{p} \cdot \boldsymbol{v} d a$

$\dot{Q}=-\int_{a_{q}} \boldsymbol{q} \cdot \boldsymbol{n} d a+\int_{V}(\boldsymbol{i} \cdot \boldsymbol{E}) d V+\int_{V}(-\dot{\Gamma}) d V$

$\dot{\Gamma}=\sum_{j=1}^{L} r^{(j)} \dot{\xi}^{(j,)}, \dot{\Pi}=\int_{V} \sum_{k=1}^{N} \mu^{(k)} \dot{\Theta}^{(k)} d V$

$\dot{\Lambda}=-\sum_{k=1}^{N} \int_{V} \nabla \cdot\left(\mu^{(k)} \boldsymbol{J}^{(k)}\right) d V$

where $\dot{U}, \dot{K}, \dot{W}, \dot{Q}, \dot{\Pi}$ and $\dot{\Lambda}$ are the internal energy rate, kinetic energy rate, work rate done by mechanical and electro-magnetic forces, external supplied heat rate, the energy production rate in chemical reaction and the energy flow introduced by the mass flow; $\mu^{(k)}$ is the chemical potential of species $k ; a=a_{q}+a_{\Phi}$ is the outer surface of the volume $V, a_{q}$ is the part of $a$ where heat flow is imputed, $a_{\Phi}$ is the part of $a$ where mass flow is imputed, $\boldsymbol{n}$ is the external normal of the surface, $\boldsymbol{p}=\boldsymbol{\sigma} \cdot \boldsymbol{n}$ is the surface traction, $\boldsymbol{q}$ is the heat flow vector, $\boldsymbol{i} \cdot \boldsymbol{E}$ is the Joule heat source strength produced by electric current; $r^{(j)}$ is the

$\dot{K}=\frac{\mathrm{d}}{\mathrm{d} t}\left(\frac{1}{2} \rho \boldsymbol{v}^{2}\right)=\nabla \cdot(\boldsymbol{\sigma} \cdot \boldsymbol{v})-\boldsymbol{\sigma}:(\nabla \otimes \boldsymbol{v})+\rho z \boldsymbol{E} \cdot \boldsymbol{v}-\boldsymbol{i} \cdot(\boldsymbol{v} \times \boldsymbol{B})+\rho \boldsymbol{f}^{(m)} \cdot \boldsymbol{v}$ 
chemical reaction heat per mass in chemical reaction $j, \Gamma$ is the total chemical reaction heat per volume in all chemical reaction, $\dot{r}>0$ for endothermal reaction and $\dot{r}<0$ for exothermal reaction.

By using Eq. (6) one has

$$
\begin{aligned}
\int_{V} & {\left[\rho\left(-\dot{\boldsymbol{v}}+\boldsymbol{f}^{(m)}+\boldsymbol{f}^{(e m)}\right) \cdot \boldsymbol{v}\right] d V+\int_{a} \boldsymbol{p} \cdot \boldsymbol{v} d a } \\
= & \int_{V}\left[\rho\left(-\dot{\boldsymbol{v}}+\boldsymbol{f}^{(m)}+\boldsymbol{f}^{(e m)}\right) \cdot \boldsymbol{v}+\nabla \cdot(\boldsymbol{\sigma} \cdot v)\right] d V \\
& =\int_{V}\left[\nabla \cdot \boldsymbol{\sigma}+\rho\left(-\dot{\boldsymbol{v}}+\boldsymbol{f}^{(m)}+\boldsymbol{f}^{(e m)}\right)\right] \cdot v d V \\
& +\int_{V} \boldsymbol{\sigma} \cdot(\nabla \otimes \boldsymbol{v}) d V=\int_{V} \boldsymbol{\sigma} \cdot(\nabla \otimes \boldsymbol{v}) d V
\end{aligned}
$$

From Eqs. (11 and 12) we get the local energy conservation equation:

$$
\begin{aligned}
\rho \dot{u} & =\sigma: \dot{\varepsilon}-\nabla \cdot \boldsymbol{q}-\dot{\Gamma}+\boldsymbol{i} \cdot \boldsymbol{E}-\nabla \cdot \sum_{k=1}^{N} \mu^{(k)} \boldsymbol{J}^{(k)}+\sum_{k=1}^{N} \mu^{(k)} \dot{\Theta}^{(k)} \\
& =\sigma_{m n} \dot{\varepsilon}-q_{m, m}-\dot{\Gamma}+i_{m} E_{m}-\sum_{k=1}^{N}\left(\mu^{(k)} \boldsymbol{J}_{m}^{(k)}\right)_{, m}+\sum_{k=1}^{N} \mu^{(k)} \dot{\Theta}^{(k)}
\end{aligned}
$$

For a general medium one form of $\sigma$ is $\sigma=\sigma^{(r)}+\sigma^{(i)}$, where $\sigma^{(r)}$ is the reversible part of $\sigma$ and $\sigma^{(i)}$ is the irreversible part of $\sigma$. For an ideal fluid $\sigma^{(r)}=-p \delta, \sigma^{(i)}=\boldsymbol{0}$ or $\sigma_{k l}=-p \delta_{k l}$, where $p$ is the fluid pressure. For an isotropic viscous fluid $\sigma=-p \delta+\beta \nabla \otimes \boldsymbol{v}$ or $\sigma_{i j}=-p \delta_{i j}+\beta v_{i, j}$, where $\beta$ is the viscous coefficient $[3,23]$.

$$
G=U-T S+p V=\sum_{k=1}^{N} \mu^{(k)} M^{(k)}, \dot{G}=-S \dot{T}+V \dot{p}+\sum_{k=1}^{N} \mu^{(k)} \dot{M}^{(k)}
$$

where $U, S, V$ and $G$ are the total energy, total entropy, total volume and total Gibbs function of the system and $M^{(k)}$ is the mass of a species $k$ respectively. From the above equation De Groot and Mazur [3] gave a specific Gibbs equation in $c^{(k)}$ :

$$
\rho \dot{u}=\rho T \dot{s}-p \dot{\bar{v}}+\rho \sum_{k=1}^{N} \mu^{(k)} \dot{c}^{(k)}
$$

where $s$ is the entropy density per mass, $\bar{v}=1 / \rho$ is the specific volume. The above equation can be directly extended to CETM system by using the theory of thermodynamics of irreversible process (TIP) $[3,23]$. Using the reversible variable $\sigma^{(r)}$ Eq. (15) can be extended to

$$
\begin{gathered}
\rho \dot{u}=\rho T \dot{s}+\sigma_{i j}^{(r)} \dot{\varepsilon}_{i j}+\rho \sum_{k=1}^{N} \mu^{(k)} \dot{c}^{(k)} \\
T=\frac{\partial \rho u}{\partial s}, \sigma_{i j}^{(r)}=\frac{\partial \rho u}{\partial \varepsilon_{i j}}, \mu^{(k)}=\frac{\partial \rho u}{\partial c^{(k)}}
\end{gathered}
$$

The formulas in second line of Eq. (16) are $N+7$ constitutive or state equations. For nonlinear medium the state equations should be obtained by experiments and material microscopic theories.

Using the first in Eq. (2) from Eqs. (13 and 16) we get the entropy equation and entropy production rate equation:

$$
\begin{aligned}
& \rho T \dot{\boldsymbol{s}}=\rho T\left(\dot{s}^{(r)}+\dot{s}^{(i)}\right)=-\nabla \cdot \boldsymbol{q}+\boldsymbol{i} \cdot \boldsymbol{E}+\boldsymbol{\sigma}^{(i)}: \dot{\boldsymbol{\varepsilon}}-\dot{\Gamma}-\sum_{k=1}^{N} \boldsymbol{J}^{(k)} \cdot \nabla \mu^{(k)} \\
& \rho T \dot{\boldsymbol{s}}^{(r)}=-T \nabla \cdot\left(\frac{\boldsymbol{q}}{T}\right)-\dot{\Gamma}, \rho T \dot{\boldsymbol{s}}^{(i)}=-\boldsymbol{q} \cdot \frac{\nabla T}{T}+\boldsymbol{\sigma}^{(i)}: \dot{\boldsymbol{\varepsilon}}+\boldsymbol{i} \cdot \boldsymbol{E}-\sum_{k=1}^{N} \boldsymbol{J}^{(k)} \cdot \nabla \mu^{(k)}
\end{aligned}
$$

\section{The entropy and evolution equations in a CETM open system}

\subsection{The entropy equation}

The Gibbs function and the Gibbs equation of the entire system for gas are respectively [1-12]
Usually in electromagnetic dynamics one introduces an electric scalar potential $\varphi$ and a magnetic vector potential A such that

$\boldsymbol{E}=-\nabla \varphi-\partial \boldsymbol{A} / \partial t, \boldsymbol{B}=\nabla \times \boldsymbol{A}$

Substituting Eqs. (18 and 4) into $\rho T \dot{s}^{(i)}$ in Eq. (17), one gets 


$$
\begin{aligned}
& \rho T \dot{s}^{(i)}=-T \dot{\eta}_{i} \frac{T_{i}}{T}+\sigma_{i j}^{(i)} v_{i, j}-\sum_{k=1}^{N} J_{j}^{(k)}\left(\bar{\mu}_{j}^{(k)}+z^{(k)} \frac{\partial A_{j}}{\partial t}\right) \\
& =-T \dot{\eta}_{i} \frac{T_{i}}{T}+\sigma_{i j}^{(i)} v_{i, j}-\sum_{k=1}^{N-1} J_{j}^{(k)}\left(\bar{\mu}_{j}^{(k)}+z^{\prime(k)} \frac{\partial A_{j}}{\partial t}\right) \geq 0 \\
& \bar{\mu}^{(k)}=\mu^{(k)}+z^{(k)} \varphi, \bar{\mu}_{j}^{\prime(k)}=\bar{\mu}^{(k)}-\bar{\mu}^{(N)}, z^{(k)}=z^{(k)}-z^{(N)}, \dot{\eta}_{i}=q_{i} / T
\end{aligned}
$$

where $\bar{\mu}^{(k)}$ is called the electrochemical potential of species $k ; q_{i}, J_{i}^{(k)}=\dot{\chi}_{i}^{(k)}$ and $v_{i, j}$ are the irreversible flows, $T_{, j} / T, \bar{\mu}_{j}^{(k)}+z^{(k)} \partial A_{j} / \partial t$ and $J^{(k)} \sigma_{i j}^{(i)}$ are the irreversible forces or vice versa. In this paper we use the notation that a comma followed by index $i$ in subscript indicates partial differentiation with respect to $x_{i}$, such as $v_{j, i}=\partial v_{j} / \partial x_{i}$. According to the irreversible thermodynamics the irreversible flows are functions of the irreversible forces. It is seen that we use $J^{(k)}$ and $z^{(k)} \varphi$ as the independent irreversible variables rather than $i^{(k)}$ and $\varphi$ themselves. At thermodynamic equilibrium $\dot{s}^{(i)}=0$, so $T_{j}=0, \partial A_{j} / \partial t=0, \bar{\mu}_{j}^{\prime(k)}=0$, $v_{i, j}=0$ and it is usual thermodynamic equilibrium conditions for electrochemical system. For quasi-stationary case $\partial \boldsymbol{A} / \partial t=\partial \boldsymbol{B} / \partial t=\boldsymbol{0}$, one can find that the role $\overline{\boldsymbol{\mu}}^{(k)}$ in electrochemistry is equivalent to $\mu^{(k)}$ in the usual chemistry. In Eq. (19) if one takes $\mu^{(k)}, z^{(k)}$ and $k=1-N$, the coefficients $M_{i j}^{(k)}$ and $N_{i j}^{(k m)}$ only $N-1$ are independent due to Eq. (3); if one takes $\mu \prime_{(j)}^{(k)}, z \prime^{(k)}$ and $k=1-(N-1)$ the coefficients $M_{i j}^{(k)}$ and $N_{i j}^{(k m)}$ are all independent. In the following sections this explanation will be continuously used.

\subsection{The evolution equation}

In usual cases the linear irreversible thermodynamics is well $[1-4,23]$, so from Eq. (19) one has

$$
\begin{aligned}
& T \dot{\eta}_{i}=q_{i}=-L_{i j} \frac{T_{j}}{T}-\sum_{k=1}^{N} M_{i j}^{(k)}\left(\bar{\mu}_{, j}^{(k)}+z^{(k)} \partial A_{j} / \partial t\right)-\kappa_{i j l} v_{j, l} \\
& \dot{\chi}_{i}^{(k)}=J_{i}^{(k)}=-M_{i j}^{(k)} \frac{T_{j}}{T}-\sum_{m=1}^{N} N_{i j}^{(k m)}\left(\bar{\mu}_{, j}^{(m)}+z^{(m)} \partial A_{j} / \partial t\right)-\gamma_{i j l}^{(k)} v_{j, l} \\
& \sigma_{i j}^{(i)}=-\kappa_{i j k} T_{, k}-\sum_{m=1}^{N} \gamma_{i j k}^{(k)}\left(\bar{\mu}_{, k}^{(m)}+z^{(m)} \partial A_{k} / \partial t\right)+\beta_{i j k l} v_{k, l}
\end{aligned}
$$

w h e re $\quad L_{i j}=L_{j i}, M_{i j}^{(k)}=M_{j i}^{(k)}, N_{i j}^{(k l)}=N_{j i}^{(k l)}=N_{i j}^{(l k)}$, $\beta_{i j k l}=\beta_{k l i j}=\beta_{j i k l}$ are constants or the functions of $T$. These coefficients are determined by experiments and the microscopic theories. For the quasi-stationary electromagnetic field, $\partial \boldsymbol{A} / \partial t=\mathbf{0}$, Eq. (20) is remarkable simplified. The second in Eq. (20) is the extended general Nernst-Planck equation in CETM system.

For the isotropic material [23] we have.
$A_{i j}=A \delta_{i j \prime}, \quad A_{i j k l}=\beta_{1} \delta_{i j} \delta_{k l}+\beta\left(\delta_{i k} \delta_{j l}+\delta_{i l} \delta_{j k}\right), \quad A_{i j k}=0$

where $A_{i j}, A_{i j k l}$ and $A_{i j k}$ are second order, fourth order and third order tensors respectively; $A, \beta_{1}$ and $\beta$ are all constants. So for an isotropic material and under quasi-stationary electro-magnetic field Eq. (20) is reduced to

$$
\begin{aligned}
& T \dot{\eta}_{i}=q_{i}=-L \frac{T_{, i}}{T}-\sum_{k=1}^{N} M^{(k)} \bar{\mu}_{, i}^{(k)} \\
& \dot{\chi}_{i}^{(k)}=J_{i}^{(k)}=-M^{(k)} \frac{T_{, i}}{T}-\sum_{m=1}^{N} N^{(k m)} \bar{\mu}_{, i}^{(m)} \\
& \sigma_{i j}^{(i)}=\beta_{1} v_{k, k} \delta_{i j}+\beta v_{i, j}
\end{aligned}
$$

where $L / T=\lambda$ is the heat conduction coefficient. $L, M^{(k)}, N^{(k m)}, \beta_{1}, \beta$ are material constants. For Stokes viscous fluid $\beta_{1}=-\beta / 3$ and $\sigma_{k k}^{(i)}=0$, i.e. only shear strain rate produces irreversible viscous stress; so $\beta_{1}=0$ and $\sigma_{i j}^{(i)}=\beta v_{i, j}$ for $i \neq j, \sigma_{i j}^{(i)}=0$ for $i=j$. The second in Eq. (22) is the extended Nernst-Planck equation for isotropic media in CETM system. Without the term $-M^{(k)} T_{, i} / T$ the second in Eq. (20) is just the usual Nernst-Planck equation [15, 27, 28]. In Nernst-Planck equation some bodies prefer $c_{j}^{(k)}$ (here $c$ is the concentration) to $\mu_{j}^{(k)}$, others are quite the reverse.

From above discussions it is seen that the combination of chemical potential $\bar{\mu}^{(k)}$ (or $\bar{\mu}^{(k)}$ ) is the fundamental variable and the $\mu^{(N)}$ is not the independent variable, but it can be obtained from the Gibbs-Duhem relation [1-3, 17-20]:

$$
\begin{array}{ll}
\sum_{k=1}^{N} \rho^{(k)} d \mu^{(k)}=-\rho s d T+d p, & \text { for ideal gas } \\
\sum_{k=1}^{N} \rho^{(k)} d \mu^{(k)}=-\rho s d T-\rho \varepsilon_{i j} d \sigma_{i j}, & \text { for general material }
\end{array}
$$

\subsection{An example}

The thermoelectricity phenomena in thermoelectricity solid are the special cases of Eqs. (20 and 22). In this cases we have a binary system $(N=2)$, one is the positive ion lattice, as species 2, another is the electron, as species 1 . The flux is measured with respect to lattice, so $\boldsymbol{J}^{(2)}=\boldsymbol{0}, \boldsymbol{J}^{(1)}=\boldsymbol{J}^{(e)}$. According to Eq. (4) $\boldsymbol{i}=\boldsymbol{z}^{(e)} \boldsymbol{J}^{(e)}$. For a quasi-statically electromagnetic field without mechanical effect Eqs. (19 and 20 or 22) are reduced to: 
$\rho T \dot{\dot{s}}^{(i)}=-T \dot{\eta}_{i} \frac{T_{i}}{T}-J_{j}^{(1)} \bar{\mu}_{, j}^{(1)}=-q_{j,} \frac{T_{j}}{T}-i_{j} \bar{\mu}_{j}^{(e)} \geq 0, \bar{\mu}^{(e)}=\varphi+\frac{\mu^{(e)}}{z^{(e)}}$

$\boldsymbol{i}=-L_{11} \frac{1}{T} \nabla \bar{\mu}^{(e)}-L_{12} \nabla\left(\frac{1}{T}\right)=-L_{11}^{\prime} \nabla \bar{\mu}^{(e)}-L_{12}^{\prime} T \nabla\left(\frac{1}{T}\right)$

$\boldsymbol{q}=-L_{12} \frac{1}{T} \nabla \bar{\mu}^{(e)}-L_{22} \nabla\left(\frac{1}{T}\right)=-L_{12}^{\prime} \nabla \bar{\mu}^{(e)}-L_{22}^{\prime} T \nabla\left(\frac{1}{T}\right)$

where $\left(L_{11}^{\prime}, L_{12}^{\prime}, L_{22}^{\prime}\right)=\left(L_{11}, L_{12}, L_{22}\right) / T$ are material constants and $L_{12}=L_{21}$ due to the Onsager reciprocal relations $[3,23]$. Equation (24) is fully consistent with Seebeck, Peltier and Thomson's theories [3,9]. It can be seen that the theory described in this paper is very simple and general. In Thomson's theory the internal points of the thermoelectric material are regular points and have continuous varying temperature or current. The junctions of different types of wire for Seebecek and Peltier effects can be considered as singular points with finite discontinuous temperature or current.

\section{The governing equations for CETM System}

At present the general governing equations for the CETM system are lack. Here we attempt to give an appropriate discussion. The first in Eq. (2) (the mass conservation equation), Eq. (6) (the momentum conservation equation) and the first in Eq. (17) (the entropy equation) can be selected as the governing equations, one has.

$\rho \dot{c}^{(k)}=-J_{j, j}^{(k)}+\dot{\Theta}^{(k)}, \quad$ or $\quad \frac{\partial \rho^{(k)}}{\partial t}=-\nabla \cdot\left(\rho^{(k)} \boldsymbol{v}\right)-\nabla \cdot \boldsymbol{J}^{(k)}+\dot{\Theta}^{(k)}$

$\sigma_{j i, j}+\rho z E_{i}+e_{i j k} l_{j} B_{k}+\rho f_{i}^{(m)}=\rho \ddot{u}_{i}$, or $\left(\sigma_{j i}+\sigma_{j i}^{M}\right)_{j}+\rho f_{i}^{(m)}=\rho \ddot{u}_{i}$

$\dot{\rho}+\rho \nabla \cdot \boldsymbol{v}=0$

$\rho T \dot{s}=-\left(T \dot{\eta}_{j}\right)_{j}-\sum_{k=1}^{N} J_{j}^{(k)}\left(\bar{\mu}_{, j}^{(k)}+z^{(k)} \frac{\partial A_{j}}{\partial t}\right)+\sigma_{i j}^{(i)} v_{i, j}-\dot{\Gamma}$

where $e_{i j k}$ is the permutation tensor, $e_{123}=e_{231}=e_{312}=1, e_{321}=e_{213}=e_{132}=-1$, others $=0$. The governing Eq. (25) is general. Usually the linear evolution Eq. (20) is often appropriate and also general. Substituting Eq. (20) into the entropy equation in Eq. (25) we get
For the isotropic material and under quasi-stationary electro-magnetic field Eq. (26) is reduced to

$$
\begin{aligned}
\rho T \dot{s}= & \left(L \frac{T_{j}}{T}+\sum_{k=1}^{N} M^{(k)} \bar{\mu}_{, j}^{(k)}\right)_{j}+\sum_{k=1}^{N}\left(M^{(k)} \frac{T_{, i}}{T}+\sum_{m=1}^{N} N^{(k m)} \bar{\mu}_{, i}^{(m)}\right) \bar{\mu}_{, i}^{(k)} \\
& +\beta v_{i, j} v_{i, j}-\dot{\Gamma}
\end{aligned}
$$

Usually $T_{j}$ and $\bar{\mu}_{, j}^{(m)}$ are small, so the term $\sum_{k=1}^{N}\left(M^{(k)} \frac{T_{i j}}{T}+\sum_{m=1}^{N} N^{(k m)} \bar{\mu}_{, i}^{(m)}\right) \bar{\mu}_{, i}^{(k)}$ including $T_{j,} \bar{\mu}_{, i}^{(k)}$ and $\bar{\mu}_{, j}^{(m)} \bar{\mu}_{, i}^{(k)}$ in equation $\rho T \dot{s}$ is small terms in higher order and in many practical cases can be neglected. In this case Eq. (27) is reduced to

$\rho T \dot{s}=\left(L \frac{T_{j}}{T}+\sum_{k=1}^{N} M^{(k)} \bar{\mu}_{, j}^{(k)}\right)_{, j}+\beta v_{i, j} v_{i, j}-\dot{\Gamma}$

Therefore for the isotropic material under the quasistationary electro-magnetic field, the general governing equations, Eq. (25) is simplified to

$$
\begin{aligned}
& \rho \dot{c}^{(k)}=-J_{i, i}^{(k)}+\dot{\Theta}^{(k)}=\left(M^{(k)} \frac{T_{, i}}{T}+\sum_{m=1}^{N} N^{(k m)} \bar{\mu}_{, i}^{(m)}\right)_{, i}+\dot{\Theta}^{(k)} \\
& \rho T \dot{s}=\left(L \frac{T_{j}}{T}+\sum_{k=1}^{N} M^{(k)} \bar{\mu}_{, j}^{(k)}\right)_{j}+\beta v_{i, j} v_{i, j}-\dot{\Gamma} \\
& \sigma_{j i, j}+\rho z E_{i}+e_{i j k} l_{j} B_{k}+\rho f_{i}^{(m)}=\rho \ddot{u}_{i,} \quad \dot{\rho}+\rho v_{i, i}=0
\end{aligned}
$$

In Eq. (29), also in Eq. (25), the unknowns $c^{(k)}, s, T, \mu^{(k)}, \sigma_{i j}, v_{i}$ are $2 N+11$, which is larger than the independent equations $N+4$, so except Eq. (29) we should add $N+7$ appropriate complement equations or constitutive (state) equations from Eq. (16).

As an example, for an ideal gas one can selects the reference state as $\left(T_{0}, p=p_{0}\right)$ and the complement equations [17-19] as:

$$
\begin{aligned}
& \rho T \dot{s}=\left[L_{i j} \frac{T_{j}}{T}+\sum_{k=1}^{N} M_{i j}^{(k)}\left(\bar{\mu}_{, j}^{(k)}+z^{(k)} \partial A_{j} / \partial t\right)+\kappa_{i j l} v_{j, l}\right]_{, i} \\
& +\sum_{k=1}^{N}\left[M_{i j}^{(k)} \frac{T_{j}}{T}+\sum_{m=1}^{N} N_{i j}^{(k m)}\left(\bar{\mu}_{, j}^{(m)}+z^{(m)} \partial A_{j} / \partial t\right)+\gamma_{i j l}^{(k)} v_{j, l}\right]\left(\bar{\mu}_{, i}^{(k)}+z^{(k)} \frac{\partial A_{i}}{\partial t}\right) \\
& +\left[-\kappa_{i j k} T_{, k}-\sum_{m=1}^{N} \gamma_{i j k}^{(k)}\left(\bar{\mu}_{, k}^{(m)}+z^{(m)} \partial A_{k} / \partial t\right)+\beta_{i j k l} v_{k, l}\right] v_{i, j}-\dot{\Gamma}
\end{aligned}
$$




$$
\begin{aligned}
& \rho T \dot{s}=\rho C_{p} \dot{T}-\alpha T \dot{p}+\rho \sum_{i=1}^{N} \bar{\mu}_{i} \dot{c}_{i}, \quad \alpha=\frac{1}{\bar{v}}\left(\frac{\partial \bar{v}}{\partial T}\right)_{p}=-\frac{1}{\rho}\left(\frac{\partial \rho}{\partial T}\right)_{p} \\
& \bar{\mu}^{(k)}=\varphi+\mu_{0}^{(k)}+R T \ln p^{(k)}=\varphi+\mu_{0}^{(k)}+R T \ln p+R T \ln c^{(k)} \\
& p=\sum_{k=1}^{N} p^{(k)}, \quad p^{(k)}=\frac{\rho^{(k)}}{M_{M}^{(k)}} R T, \quad p \bar{v}=R T, \quad \sigma_{i j}=-p \delta_{i j}
\end{aligned}
$$

In Eq. (30) $\alpha$ is the dilatation coefficient, $C_{p}$ is the specific heat per mass.

Substituting the first of Eq. (30) into the second of Eq. (29), we get the energy equation or the temperature (heat) equation for an ideal gas:

$$
\rho C_{p} \dot{T}-\alpha T \dot{p}+\rho \sum_{i=1}^{N} \bar{\mu}_{i} \dot{c}_{i}=\left(L \frac{T_{j}}{T}+\sum_{k=1}^{N} M^{(k)} \bar{\mu}_{, j}^{(k)}\right)_{j}+\beta v_{i, j} v_{i, j}-\dot{\Gamma}
$$

where the chemical reaction heat $\Gamma$ is a part of temperature equation and the electric potential is included in the electrochemical potential.

In order to solve the practical engineering problems the boundary conditions are needed. The boundary conditions in CETM system are complex and different in various cases and it should be discussed case by case.

The rest of this section some comments will be given.

Current theories in literatures for CETM system are all approximate theories. The general governing Eq. (29) can be used to estimate which elements are neglected in these present theories and can also be regarded as reference theory to improve them. When one discusses the gradual failure of components and cells the temperature effects may be important which is included in Eq. (29).

In electric battery the general governing Eq. (29) can directly apply to the liquid electrolyte region. Natural and forced convection and the diffusion, the electric, chemical, temperature and mechanical effects are all taken into account. In ion-exchange membranes, such as fuel cells, the Chlor-Alkali process, and water electrolysis etc., in the electrode region the ion/molecule reaction needs absorb heat from the environment or release heat to the environment. The molecule (or atom) and ion can be considered as two components of the system. But in existing theories these effects are all neglected [29-31]. In the liquid electrolyte region the temperature effect is also neglected. In [15] Loret and Simões, discussed the deformation, diffusion, mass transfer and growth in multispecies multi-phase biological tissues detailely. They proposed that the mass change due to the tissue growth is produced by two physical phenomena, namely diffusion of the species through the solid skeleton, and mass transfer or organic growth supplied by the environment, such as foods through certain chemical reaction or "organic work", rather than chemical reaction heat $\Gamma$. In this case $\dot{\Theta}^{(k)}$ in Eq. (2) should be understood as the mass growth of the biological tissues, and in Eq. (11) $\dot{\Gamma}=0$ in formula $\dot{Q}$, but in formula $W$ a term "organic work rate" produced by foods etc. should be added. However the biological processes and the mechanical loadings correspond to largely different time scales, so in the energy rate Eq. (11) the biological growth rate can often be neglected. But the initial states solving the differential governing equations corresponding to different time are different due to the biological growth. The electromagnetic effects may also be fully taken into account in the biological processes.

\section{Conclusions}

In this paper a complete energy equation including the variations of the structure energy and the reaction heat are derived for coupled heterogeneous chemo-electrothermo-mechanical diffusive systems. Applying this energy equation, the corresponding entropy and entropy production rate equations are obtained. The evolution equations derived from the entropy production rate equations are fully consistent with current classical theories. The extended Nernst-Planck equation in CETM system is obtained. Some interesting questions are proposed. The present theory modifies and simplifies existing theories and can be used as the foundation to improve the practical engineering theories. In appendix we also discuss interdiffusion problems in the solids with vacancies shortly as a complement of the continuum diffusion. The theory and opinions in this paper should be tested in practice.

\section{Compliance with ethical standards}

Conflict of interest The authors declare that they have no conflict of interest.

\section{Appendix: The interdiffusion problems in solids}

Other than fluids, in solids diffusion is the only way to move atoms. So for a solid generally $\boldsymbol{v}=\mathbf{0}$ and $\rho=$ const. However the Kirkendall effect shows that the interface $S_{\text {int }}$ (the inert marker) between two metals with different diffusion rates of the metal atoms can be moved. This interdiffusion phenomenon in a diffusion couples of binary system is related to vacancies in noncontinuum media and is 
different with above textuary theory. For the continuum media in the mass conservation Eq. (2) $v$ is the barycentric velocity, but in the interdiffusion problems the interface $S_{\text {int }}$ movement speed $\overline{\boldsymbol{v}}$ is introduced by the vacancy diffusion mechanism in the metallic materials.

Let $\pi^{(k)}$ be an atomic concentration or the number of atom particles per volume of species $k$. Let a metal $k^{(1)}$ with a kind of actual atom particle diffusion flow $\boldsymbol{J}_{R}^{(1)}=\pi^{(1)} \overline{\boldsymbol{v}}^{(1)}$ in a fixed coordinate system, be in the left of $S_{\text {int }}$ and a metal $k^{(2)}$ flow $\boldsymbol{J}_{R}^{(2)}=\pi^{(2)} \overline{\boldsymbol{v}}^{(2)}$ be in the right of $S_{\text {int }}$. Similar to Eqs. (2) and (23) under isothermal condition the mass diffusion flow $\boldsymbol{J}^{(k)}$ relative to interface $S_{\text {int }}$ is

$$
\begin{aligned}
& \boldsymbol{J}^{(k)}=\pi^{(k)}\left(\boldsymbol{v}^{(k)}-\overline{\boldsymbol{v}}\right), \quad \boldsymbol{J}^{(k)}=\sum_{m=1}^{2} N^{(k m)} \nabla \mu^{(m)} \\
& \boldsymbol{J}_{R}^{(k)}=\pi^{(k)} \boldsymbol{v}^{(k)}=\pi^{(k)} \overline{\boldsymbol{v}}+\boldsymbol{J}^{(k)}, \quad k=1,2
\end{aligned}
$$

where $N^{(\mathrm{km})}$ is the diffusion coefficient for isotropic materials. The total number of atom particles in two metals should be constant. So one has

$$
\boldsymbol{J}_{R}^{(1)}+\boldsymbol{J}_{R}^{(2)}=\sum_{m=1}^{2}\left(N^{(1 m)}+N^{(2 m)}\right) \nabla \mu^{(m)}+\left(\pi^{(1)}+\pi^{(2)}\right) \overline{\boldsymbol{v}}=\mathbf{0}
$$

For the isotropic material $N^{(i m)}=-D^{(i)}$ if $m=i$; $N^{(i m)}=0$, if $m \neq i$, where $D^{(i)}$ is diffusion coefficient of metal $k^{(i)}$, so Eq. (33) becomes

$$
\begin{aligned}
& \boldsymbol{J}_{R}^{(1)}+\boldsymbol{J}_{R}^{(2)}=-\left(D^{(1)} \nabla \mu^{(1)}+D^{(2)} \nabla \mu^{(2)}\right)+\left(\pi^{(1)}+\pi^{(2)}\right) \overline{\boldsymbol{v}}=\mathbf{0} \\
& \overline{\mathbf{v}}=\frac{1}{\pi^{(1)}+\pi^{(2)}}\left(D^{(1)} \nabla \mu^{(1)}+D^{(2)} \nabla \mu^{(2)}\right)
\end{aligned}
$$

Equation (3) is equivalent to the Darken equation.

Okino considered that the Darken first equation is inconsistent with the fundamental theory in mathematics and proposed that: "the invalid Darken theory and the misjudged descriptions in existing text books should be revised or deleted as soon as possible" $[32,33]$. He proposed a "new diffusion flux $\boldsymbol{J}_{R}(t, x, y, z)=-D \nabla C(t, x, y, z)-\boldsymbol{J}_{0}(t)$ ", "where $\boldsymbol{J}_{0}(t)$ is defined as a vector on $S$, it is relevant to the material inflow caused by a material source on $S$ " (where $S$ is a boundary surface surrounding the discussed volume). Though $\boldsymbol{J}_{R}^{(k)}$ in (32-34) contains also a vector $\pi^{(k)} \overline{\boldsymbol{v}}(t)$, (or $\overline{\boldsymbol{v}}(t)$ when one discusses the atomic fraction,), but it represents the speed of the interface introduced by the vacancy diffusion mechanism rather than initial condition or"a vector on $S^{\prime \prime}$. In other words, Darken equation is rationality, though it may not be fully exact. The interdiffusion problem is related to vacancies, belong to the noncontinuum physics. It cannot use the mathematical model established on the basis of continuum media.

\section{References}

1. Kuang Z-B (2015) Energy and entropy equations in coupled nonequilibrium thermal mechanical diffusive chemical heterogeneous system. Sci Bull 60: 952-957. Corrigenda: Sci. Bull. (2018) 63: 732

2. Kuang Z-B (2019) A general theory for heterogeneous coupled chemical reaction-thermal diffusion systems. Chem Phys Lett 715:268-272

3. De Groot S, Mazur RP (1969) Nonequilibrium Thermodynamics, Dover Publications. INC, New York

4. Demirel Y (2009) Thermodynamically coupled heat and mass flows in a reaction-transport system with external resistances. Int J Heat and Mass transf 52:2018-2025

5. Deen WM (1998) Analysis of transport phenomena. Oxford University Press, Oxford

6. Schmidt LD (2005) The engineering of chemical reactions. Oxford University Press, New York

7. De Zarate JMO, Sengers JV, Bedeaux D, Kjelstrup S (2007) Concentration fluctuations in nonisothermal reaction-diffusion systems. J Chem Phys 127:034501

8. Hu S, Shen S (2013) Non-equilibrium thermodynamics and variational principles for fully coupled thermal-mecganical-chemical processes. Acta Mech 224:2895-2910

9. Goldsmid HJ (2010) Introduction to thermoelectricity. Springer, Heidelberg

10. Swaminathany N, Qu J, Sun Y (2007) An electrochemomechanical theory of defects in ionic solids. ITheory. Philos Mag 87:1705-1721

11. Maier J (2004) Physical chemistry of ionic materials, ions and electrons in solids. Wiley, Hoboken

12. Singhal SC, Kendall K (2003) High temperature solid oxide fuel cells fundamentals design and applications. Elsevier, Amsterdam

13. Liu M (1997) Distributions of charged defects in mixed ionicelectronic conductors, part I, general equations for homogeneous MIECs. J Electrochem Soc 144:1813-1834

14. Loret B, Simões FMF (2005) Mechanical effects of ionic replacements in articular cartilage: Part I-the constitutive model, Biomech Modeling. Mechanobiology 4: 63-80 Part II: Simulations of successive substitutions of $\mathrm{NaCl}$ and $\mathrm{CaCl}_{2} \cdot 4: 81-89$

15. Loret B, Simões FMF (2005) A framework for deformation, generalized diffusion, mass transfer and growth in multi-species multi-phase biological tissues. Eur J Mech A/Solids 24:757-781

16. MoyneMurad CMA (2002) Electro-chemo-mechanical couplings in swelling clays derived from a micro/macro-homogenization procedure. Int J Solids Struct 39:6159-6190

17. Weinhold $F(2009)$ Classical and geometrical theory of chemical and phase thermodynamics. Wiley, Hoboken

18. Denbigh KG (1981) The principoles of chemical equilibrium with applications in chemistry and chemical engineering (fourth ediortionm). Cambridge University Press, London

19. Jiang L-C, He G-P, Sun Y-H, Zuo X-X, Ma G-Z (2013) Physical chemistry, 4th edn. Higher education Press, Beijing ((in Chinese))

20. Gao Z-D (2006) Fundations of chemical thermodynamics. Beijing University Press, Beijing ((in Chines))

21. Darken IS (1948) Diffusion, mobility and their interrelation through free energy in binary metallic system. Trans AIME 175:184-201

22. Darken LS (1967) Thermodynamics of binary metallic solutions. Trans Met Soc AIME 239(80-89):90-96

23. Kuang Z-B (2002) Nonlinear continuum mechanics. Shanghai Jiaotong University Press, Shanghai ((in Chinese))

24. Pao YH (1997) Electromagnetic force in deformable continua. In: Nemat-Nasser S (ed) Mechanics today. Pergamon Press, Oxford, pp 209-305

25. Zhen-Bang K (2014) Theory of Electroelasticity. Berlin, Springer, (2013) Shanghai Jiaotong University Press, Shanghai 
26. Landau LD, Lifshitz EM (1960) Electrodynamics of continuum Media. Pergamon Press, Oxford

27. Strathmann $\mathrm{H}$ (2004) lon-exchange membrane separation processes, 1 st edn. Elsevier, Amsterdam

28. Moshtarikhah S et al (2017) Nernst-planck modeling of multicomponent ion transport in a nafion membrane at high current density. J Appl Electroche 47:51-62

29. Dao T-S et al (2012) Simplification and order reduction of lithium-ion battery model based on porous-electrode theory. J Power Sour 198:329-337

30. Cha Q (2002) Introduction to kinetics of electrode process. Science press, Beijing (in Chinese)
31. Reddy RD, Cameselle C (2009) Electrochemical remediation technologies for polluted solids Sediments and Groundwater. Wiley, Hoboken

32. Okino T (2012) Theoretical evidence for revision of fickian first law and new understanding of diffusion problems. J Mod Phys 3:1388-1393

33. Okino T (2013) Ending of darken equation and intrinsic diffusion concept. J Mod Phys 4:1495-1498

Publisher's Note Springer Nature remains neutral with regard to jurisdictional claims in published maps and institutional affiliations. 